\title{
Evidence for the Embodiment of the Automatic Approach Bias
}

\author{
Johannes Solzbacher $^{1}$, Artur Czeszumski ${ }^{1}$, Sven Walter ${ }^{1}$, Peter König ${ }^{1,2}$ \\ ${ }^{1}$ Institute of Cognitive Science, Universität Osnabrück, Osnabrück Germany \\ ${ }^{2}$ Department of Neurophysiology and Pathophysiology, University Medical Center Hamburg-Eppendorf, \\ Germany
}

\begin{abstract}
Author Note
This project was funded by the Deutsche Forschungsgemeinschaft (DFG, German Research Foundation) - project number GRK-2185/1 (DFG Research Training Group Situated Cognition).
\end{abstract}

A stable link to a repository with all data and analysis will be made available in a later version of the revision. Until that point, there will be a placeholder in place of the link in the manuscript.

This study was not preregistered.

Word Count: 6013

Number of Figures: 5

Number of Tables: none

Corresponding Author:

Johannes Solzbacher

Address: Institute of Cognitive Science

Wachsbleiche 27 (building 50)

D-49090 Osnabrück

Phone: +49 17657974299

Email: jsolzbacher@uos.de 


\section{Abstract}

Tendencies of approach and avoidance seem to be a universal characteristic of humans. Specifically, individuals are faster in avoiding than in approaching negative stimuli and they are faster in approaching than in avoiding positive stimuli. The existence of this automatic approach/avoidance bias has been demonstrated in many studies. Furthermore, this bias is thought to play a key role in psychological disorders like drug addiction and phobias. However, its mechanisms are far from clear. Theories of embodied cognition postulate, that the nature of gestures play a key role in this process.

To shed light on the role of the involved gesture we employed a $2 \times 2$ factorial design with two types of stimuli. Participants had either to approach positive and avoid negative stimuli (congruent conditions) or to avoid positive stimuli and approach negative stimuli (incongruent conditions). Further, they responded either with a joystick or a button press on a response pad.

Participants reacted faster in congruent conditions, i.e., avoiding negative stimuli and approaching positive stimuli, then in incongruent conditions. This replicates the known approach and avoidance bias. However, directly analyzing the button press condition participants revealed no reaction time advantage for congruent trials compared to incongruent trials. In contrast, in the joystick condition participants were significantly faster performing congruent reactions than incongruent reactions.

This interaction, a significant reaction time advantage, when the response is enacted by moving a joystick towards or away from the body gives evidence that approach-avoidance tendencies have a crucial bodily component.

Embodiment | Approach-Avoidance Task | Cognition | Automatic Approach/Avoidance Bias Correspondence:.jsolzbacher@uos.de 


\section{Contribution to the Field Statement}

Behavior of approach and avoidance are two of the most primal and natural forms of reaction in the spectrum of human reaction-behavior. Unsurprisingly, it is at least partly automatic and humans have a bias to avoid things that they fear or dislike and to approach things that they like or want. Currently the modification of this automatic approach/avoidance bias is used in therapy of substance use disorder, depression, phobia and other psychological disorders that are related to approach or avoidance. The current study suggests and provides evidence that automatic behavior of approach and avoidance carries a crucial bodily component. To show this, we systematically compared two different approachavoidance task setups, in which participants had to react to positive or negative stimuli. However, in one setup participants reacted using a joystick and a pulling/ pushing-gesture while in the other setup they react with gestures that have no significant bodily meaning (mere button presses). We show that the bodily amplification makes for a crucial difference in reaction times. Looking forward, the bodily amplification of approach/avoidance behavior might be the basis to foster known ways of treating substance use disorder or other psychological disorders connected to approach/avoidance.

\section{Introduction}

Behaviors of approach and avoidance are two of the most primal and natural ways to generally act and react to encounters of any kind in our world. We naturally approach known friends when we meet them on the street and avoid obscure alleys in the night. In our everyday life we generally tend to approach certain things rather than avoiding them, mostly if we associate the encounter with something positive to happen. Likewise, we avoid certain things rather than approaching them, mostly if we expect something negative from the encounter. Those general behavioral tendencies can in certain contexts be observed in connection with psychological disorders like drug addiction (Ernst et al. 2014) or phobia (Rinck \& Becker 2007) and their modification is used in therapy and treatment (Hertel and Mathews 2011; Wiers et al. 2011). Thus, understanding approach and avoidance behavior might not only be 


\section{EMBODIEMENT OF THE AUTOMATIC APPROACH BIAS}

indispensable for a better understanding of cognition in general but particularly helpful for psychological and therapy-related reasons.

One way to study and measure an individual's tendency to approach or avoid certain types of stimuli rather than others is using what is called an approach-avoidance task. In this reaction time based setup individuals are instructed to either push away or pull towards them specific cues like words (Chen and Bargh 1999) or pictures (Bradley et al. 2008) for example according to their valence (Phaf et al. 2014) as fast as they can. Here, a general individuals' tendency to approach positive stimuli rather than avoiding them and to avoid negative stimuli rather than approaching them is also reflected in their reaction times. Similar effects have been shown for individuals suffering from substance use disorder like heavy drinkers (R. W. Wiers et al., 2009), alcohol abuse disorder (Wiers et al. 2011; Wiers et al. 2014), heroin addicts (Y. Zhou et al. 2012), smokers (Bradley et al. 2008; Wiers et al. 2013; Mühlig et al. 2016) and cannabis users (Cousijn et al., 2011). All these groups approach drug-related cues faster than neutral cues or faster than healthy controls. But the effect extends even further: Spider phobics are faster in avoiding spider-related stimuli than in avoiding neutral cues (Rinck \& Becker 2007) and similar effects hold for socially anxious people (Heuer et al. 2007) and excessive online gamers, who approach gaming-related stimuli faster than neutral cues (Jeromin et al. 2016; Z. Zhou et al. 2012). Healthy individuals seem to exhibit an approach bias for chocolate (Dickson et al. 2016) and food in general (Booth et al. 2018; Schroeder et al. 2016). Generally, approach-avoidance tasks show that we are faster in avoiding what we fear or dislike and in approaching what we want or like. This reaction-time-based effect is called an automatic approach/avoidance bias.

While approach and avoidance tendencies might be a rather universal characteristic, understanding their underlying mechanisms is particularly important for individuals struggling with substance use disorder or other forms of psychological disease. The automatic approach bias can be modified by means of implicit approach-avoidance tasks in which participants are retrained to push certain (e.g. alcohol-related) cues away while pulling others (mostly neutral cues) towards themselves. Even after only short training periods, this method called cognitive bias modification has profound 


\section{EMBODIEMENT OF THE AUTOMATIC APPROACH BIAS}

effects (Wiers et al. 2011) — for instance, in heavy drinkers it reduces alcohol craving, arousal ratings of alcohol pictures, and relapse rates, and even leads to a reduction in cue-evoked activation in the amygdala (Wiers et al. 2015). Similar effects have been proclaimed for anxiety disorders (MacLeod and Mathews 2012; Stevens et al. 2018), depression (Koster and Hoorelbeke 2015; Tendolkar et al. 2019), excessive online gaming (Rabinovitz and Nagar 2015), obesity (Kakoschke et al. 2018; Mehl et al. 2019), phobias (Fox et al. 2015), and smoking (Mühlig et al. 2016). However, the therapeutic application of modifying cognitive biases of approach and avoidance is still a rather recent development.

Exploring its mechanisms and developing new ways to potentiate the effect seems to be crucial in pushing forward this approach to therapy. In cognitive bias modifications the retraining of the biases relies on a setup similar to the approach-avoidance task and is therefore usually performed with a joystick. One way to get more insight into the mechanisms of approach-avoidance biases and their therapeutic modification is to investigate the role of the involved bodily gesture, i.e., of pushing something away and pulling something towards oneself.

In recent years and decades, an upsurge of embodied theories has been seen in all sorts of areas of cognitive science (e.g. Clark 1998; Gallagher 2006; Engel et al. 2013; Goldman 2013). Most of those theories depart from traditional accounts that focus solely on abstract, amodal, symbolic information processing as the basis for cognitive processes (e.g. Marr 1982; Fodor 1975; Fodor 1981). This development has led to a better understanding of the way in which cognitive processing is shaped by the structure of our body, our bodily actions and/or our interaction with the environment, such as pushing or pulling a joystick. While it has long been known that appraisals can be modified by performing actions associated with a particular valence (Strack et al. 1988), the exact relationship between an individual's perceptual and motor representations and the associated approach and avoidance tendencies is still far from clear. One particularly promising embodied account to explain the association between abstract concepts like approach and avoidance and sensorimotor patterns like 


\section{EMBODIEMENT OF THE AUTOMATIC APPROACH BIAS}

pushing and pulling, and thereby to an explanation of the automatic approach bias, is the 'biological meaning model' presented by Fridland and Wiers (2018).

According to the biological meaning model ${ }^{1}$ (Fridland and Wiers, 2018) both the automatic approach bias and cognitive bias modification can be explained by the fact that our body (morphology, physiology, shape and functions) is made up in a certain evolutionary meaningful way. In particular, the centers of our bodies happen to house the most vulnerable organs. Thus, it seems reasonable for humans to have developed a disposition to only allow trustworthy objects to come close to it. Since those vulnerable regions are crucial to protect, along the same lines it seems reasonable for humans to also have developed a disposition to keep dangerous or harmful objects away from our center. Consequently, according to this model pulling something towards us naturally indicates that it is positive (i.e. trustworthy, nourishing etc.) and pushing something away naturally indicates that it is negative (i.e. dangerous, disgusting etc.).

Unfortunately, while such an embodied account seems prima facie plausible and might arguably be not only of theoretical, but also of clinical importance, the details are still far from clear. Amongst other problems, there appears to be one fundamental worry that makes it difficult to argue despite the current popularity of embodied approaches to cognition in general and to the automatic approach bias and cognitive bias modification in particular - that they are in fact embodied at all. A grave problem for any embodied account is that at least one study apparently found an automatic approach bias using an approach-avoidance task that just required subjects to press the $\uparrow$ - and $\downarrow$-keys on a keyboard (Peeters et al. 2012). If an automatic approach bias can occur in the absence of any (significant) bodily approach or avoidance movement, no straightforward embodied account can offer an adequate explanation of the underlying mechanisms.

\footnotetext{
${ }^{1}$ Besides the Biological Meaning Model Fridland and Wiers also offer a second embodied account. It is called 'Sensory Motor Hypothesis' and reflects the idea of approach and avoidance as learned sensory motor loops.
} 


\section{EMBODIEMENT OF THE AUTOMATIC APPROACH BIAS}

In this paper we try to shed light on this problem and contribute to the current discussion by providing important evidence that the automatic approach bias is indeed embodied or at least carries an embodied component, while at the same time casting doubt on the claim that an automatic approach bias can also be detected by means of button press approach-avoidance tasks. For this we conducted a systematic comparison between two kinds of approach-avoidance tasks. One approach-avoidance task involved a decidedly body-related gesture representing approach/avoidance (pushing vs. pulling using a joystick), the other involved a response movement that is arguably neutral in terms of bodily significance at the very least in the context of approach and avoidance (pressing a button). We used generally positive and negative pictures as stimuli and healthy subjects as trial group. In line with previous research, we expected to find an automatic approach bias for the joystick approach-avoidance task. In light of the results found by Peeters et al. (2012) we also reckoned with the possibility of finding an automatic approach bias for the button press approach-avoidance task. However, given the recent upsurge of embodied approaches to cognition in general and the promising theories about embodiment in terms of the automatic approach bias in particular (Fridland and Wiers 2018), we expected that even if an automatic approach bias were to occur in pure button press approach-avoidance tasks at all, actually performing the bodily approach or avoidance movements in the joystick approach-avoidance task should potentiate the effect. If we found automatic approach biases in both conditions, and if using a joystick instead of pressing buttons would indeed potentiate the effect, we were ready to argue, that the gesture of approach and avoidance that is present in the joystick but not in the button press plays a crucial role for the explanation of automatic approach biases. 


\section{Methods}

\section{Participants}

51 participants (17 male, all right-handed, mean age of 24 years, standard deviation of $\sim 3.6$ years) participated in the experiment. All participants gave written informed consent before the start of the experiment and received either $10 €$ or course credits in exchange for their participation. All participants had normal or corrected to normal vision and were advanced or native speakers of English. All instructions were shown and explained in English. The ethics committee of Osnabrück University approved the study.

\section{General Apparatus}

We presented all stimuli on a 24" LCD monitor (BenQ XL2420T; BenQ, Taipeh, Taiwan) with a refresh rate of $114 \mathrm{~Hz}$. The joystick used for the joystick approach-avoidance task (Logitech Gaming Extreme 3D Pro Joystick USB PC, Black, Silver) was directly connected to the computer screen. The 


\section{EMBODIEMENT OF THE AUTOMATIC APPROACH BIAS}

response pad used for the button press approach-avoidance task (Black Box Toolkit USB response pad, info@blackboxtoolkit.com) was connected to the computer with an extension cable for USB. Matlab's Psychtoolbox V3 (Kleiner et al. 2007) (r2017a; MathWorks Company) enabled us to record reaction times of both the pushing/pulling movements as well as the button presses on the response pad. Participants positioned themselves in front of the screen such that they could naturally and effortlessly hold and use both devices. This was important to avoid a bias by e.g. making it hard to push the joystick for participants with short arm length because the device would be too far away. The subjects were instructed to autonomously change between devices when they were requested to do so on the screen. We used MATLAB and R to analyze all data. Both analysis and raw data are available online at (LINK TO BE INSERTED).

\section{Stimuli}

The experiment consisted of four different approach-avoidance tasks for each subject (see below for details). There were two different devices (joystick, response pad) and two different instructions (congruent, incongruent), where participants had to pull positive pictures and push negative pictures for the congruent condition and pull negative pictures and push positive pictures for the incongruent condition. Instruction and device yield by combination the four different blocks. The first two blocks were always performed with the same device to minimize switches between devices. Both stimuli and block order were randomized over subjects. As stimuli we used 88 fullcolored images from the International Affective Picture System (IAPS) (Lang et al.

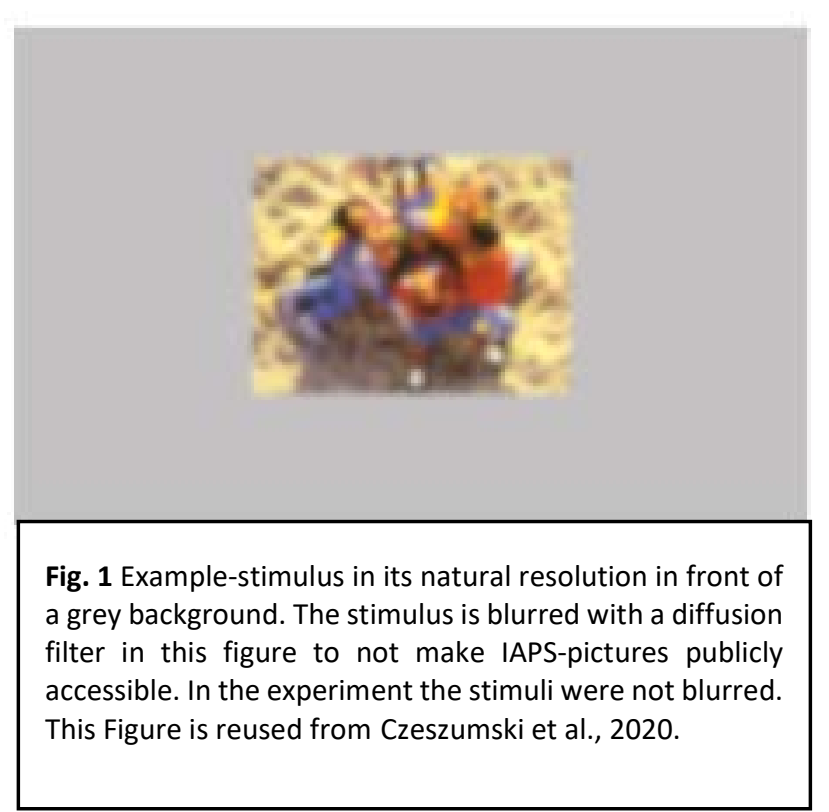




\section{EMBODIEMENT OF THE AUTOMATIC APPROACH BIAS}

1997). For reasons of comparability we used a stimulus set identical to one being used by other studies and being accessible for other researchers (Kaspar et al. 2015; Czeszumski et al. 2020). Half of the images had a valence rated below 3 (IAPS scale) and served as negative stimuli. The other half had valence ratings above 7.2 and served as positive stimuli. To prevent the images from blurring, we presented all of them in their native resolution of 1024 x 768 pixels on a grey background (RGB values: 182/182/182), centered in the middle of the screen (resolution of $1920 \times 1080$ pixels, Figure 1).

\section{Procedure and Design}

Participants were randomly assigned to one of four groups (A, B, C, D) differing in block order. For convenience we maintained only one switch of devices for each group, while still counterbalancing for both device-order and instruction-order. This yields the following four different sequences $(\mathrm{J}=$ Joystick, $\mathrm{R}=$ Response pad, I = Incongruent, $\mathrm{C}=$ Congruent)
A: $\quad \mathrm{JC}-\mathrm{JI}-\mathrm{RC}-\mathrm{RI}$
B: $\quad$ JI - JC - RI - RC
$\mathrm{C}: \quad \mathrm{RC}-\mathrm{RI}-\mathrm{JC}-\mathrm{JI}$
D: $\quad$ RI - RC - JI - JC

In each block, participants faced a sequence of 44 images of different valence ( 22 positive, 22 negative images). The first four images in each block were test trials and were excluded from analysis. All images were shown twice: Once in the first two blocks, and once in the following two blocks. Stimulus order was randomized both between blocks and between subjects. Due to a technical issue the first eight subjects received the same pseudo random stimulus order. As soon as an image was presented, the participants had to respond to the valence of the image with either the joystick or the response pad, using their dominant hand. In line with previous button press approach-avoidance setups (Peeters et al. 2012) and recent analysis of the field (Fridland and Wiers 2018) we assumed that the up button ( $\uparrow$ ) can naturally be interpreted as pointing away from the participants. Thus, pressing the up button on the 


\section{EMBODIEMENT OF THE AUTOMATIC APPROACH BIAS}

response pad corresponded to pushing the joystick (expressing avoidance). Conversely, we assumed that the down button can naturally be interpreted as pointing towards the subject, thus pressing the down button on the response pad corresponded to pulling the joystick (expressing approach). Participants in the congruent task condition thus had to pull the joystick towards them or press the down button (approach) whenever a positively valanced image was shown, and push the joystick away or press the up button (avoidance) whenever a negatively valanced image was shown. In the incongruent task condition, participants had to act reversely, meaning they had to approach the negatively valanced images and avoid the positively valanced ones. All subjects were instructed to respond as quickly and as accurately as possible. It was not possible to rectify and correct response mistakes. In line with Peeters et al. (2012), we instructed the participants to press the corresponding button on the response pad 3 consecutive times. We recorded all button presses and evaluated the data using the first button press only. For the sake of completeness and in line with Peeters et al., (2012) we repeated all data analysis also using the third button press without finding significant differences in the results. In line with the inability to correct errors in the joystick task, responses in which different buttons were pressed within one trial were treated as errors.

In line with prior research (e.g. Czeszumski et al. 2020), we used a "zooming-effect" to enhance the impression of a movement of approach or avoidance, respectively. While moving the joystick or when pressing the button on the response pad for the third time, the image changed in size in a way that was supposed to enhance the impression of approach or avoidance. In avoidance-conditions (push

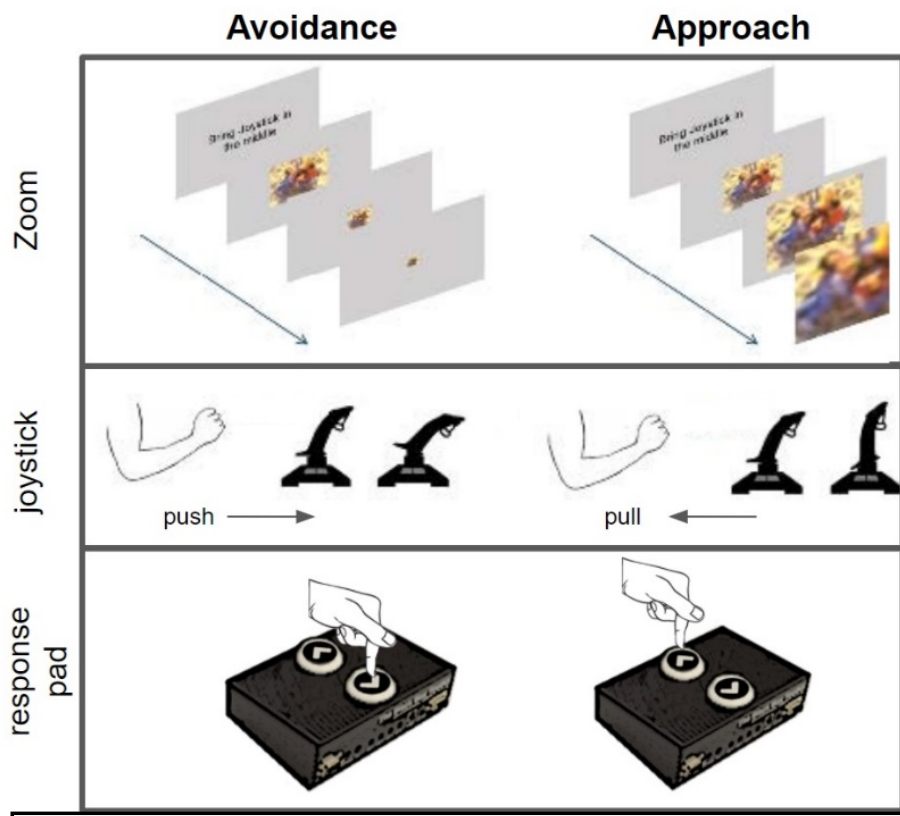

Fig. 2 The Upper part shows the Zoom-effect for both directions. The lower parts show approach and avoidance reaction for both devices (Joystick and Response pad). This Figure is partly reused from Czeszumski et al. 2020.

joystick, press the up button on the response pad) the image presented smoothly decreased in size. 


\section{EMBODIEMENT OF THE AUTOMATIC APPROACH BIAS}

Conversely, in approach conditions (pull joystick; press the 'down button on the response pad) the image presented smoothly increased in size (Figure 2). This zoom feature of the approach-avoidance tasks was programmed in MATLAB's Psychtoolbox V3 (r2017a; MathWorks Company) and taken directly from (Czeszumski et al. 2020) with only few adjustments made in the code. Participants were instructed to push or pull the joystick to its limit. Generally, participants had control over when the next stimulus would appear by pressing the index finger button on the joystick or the left button on the response pad. Instructions were repeatedly shown between stimuli to ensure that participants always were aware of the current instruction. Between blocks subjects took a break of at least 15 seconds.

\section{Results}

\section{Accuracy \& error-trial exclusion}

We gathered data from 51 participants * 176 trials, yielding a total of 8976 data points. Each block started with 4 test trials, which were excluded from analysis $(51 * 4 * 4=816$ test trials). 4 Subjects did not notice a change of instructions between blocks and thus had an accuracy of under $75 \%$. Since those subjects had to be instructed again, they were completely excluded from analysis to avoid instruction biases in the data $(4 *(176-16)=640$ additional trials excluded $)$. For the remaining data (7520 trials) we calculated an accuracy of $96,3 \%$. Thus, subjects made a low amount of errors $(3.7 \%)$. This indicates that instructions were clear, and all participants remaining in the analysis followed them with high accuracy. Therefore, we excluded all remaining error trials from any further analysis.

\section{Pre-processing}

To make data accessible and to prepare for statistical analysis in general we decided to perform a pre-analysis observation of the data. First, we divided all data into the four different conditions (joystick-incongruent, joystick-congruent; button press-congruent; button press-incongruent). The raw data showed a right-skewed distribution for all conditions and generally not a lot of variance to the left side of the mean. This is to be expected because the fastest human reaction times have been known to 


\section{EMBODIEMENT OF THE AUTOMATIC APPROACH BIAS}

be around $150 \mathrm{~ms}$. Consequently, for values lower than that there is not much space for variance. In contrast, sometimes people needed 3, 4 or even more seconds to react to a picture, allowing for large variance to the right side of the distribution. After the experiment some subjects reported that they experienced a 'brain freeze' for negative pictures they did not want to pull towards them, meaning they came into a state of cognitive dissonance in which they did not want to react at first but then remembered they had to, which led to a delayed reaction. This might be one explanation, among others, for the existence of a few very long reaction times. Discarding those trials, as they are not artifacts but true reactions, would have led to a loss of valuable information. Thus, a first observation of the data, yielded several challenges before we could proceed to the main statistical analysis.

To account for the right-skewed distribution and actions by the participants not related to a response we took further steps of preprocessing. First, all trials with reaction times below $150 \mathrm{~ms}$ were identified as not intentional responses but signals unrelated to task and visual stimulation and were discarded as such (24 trials were discarded this way). Those trials mostly occurred for the joystick condition (all but one trial) and can be explained by subjects putting their hands and therefore weight on the joystick after bringing it back to a neutral position. When subjects than made the next picture appear, the program detected a response immediately because the joystick was in a pulled position already when the picture appeared. Second, for outliers that corresponded to very long reaction times, we decided to use a 4-standard-deviation-winsorizing procedure to adjust the distribution without losing valuable information (reaction times of 65 trials were shifted that way, 33 joystick trials, 32 buttonpress trials). Since the data still showed a skewed distribution after winsorizing, we log-transformed it before further analysis (using natural logarithm) approximating a gaussian distribution. Eventually the 


\section{EMBODIEMENT OF THE AUTOMATIC APPROACH BIAS}

data showed a normal distribution and all aspects of the reaction times were treated satisfactory, thus we continued with the main analysis.

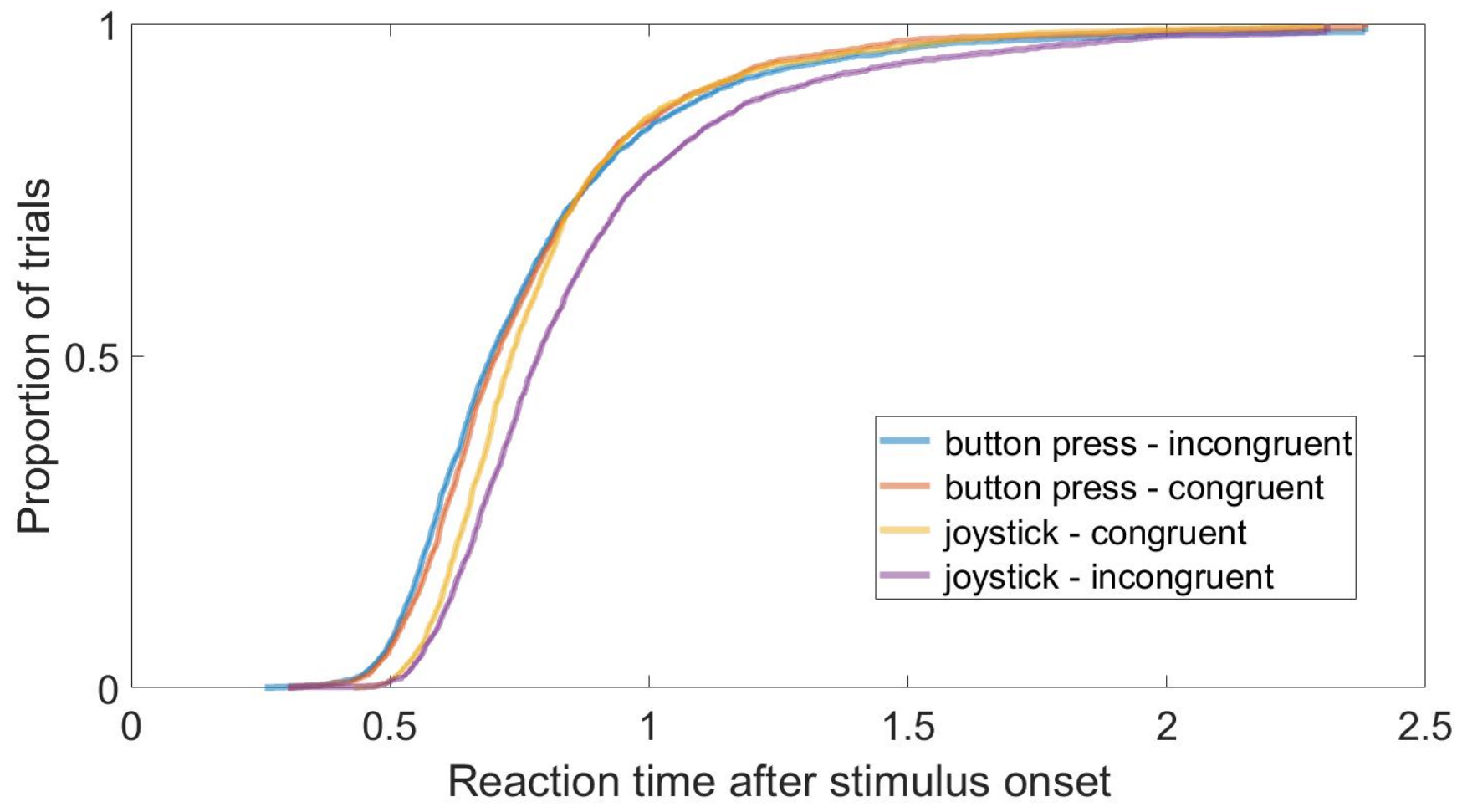

Fig. 3 Cumulative Distribution Function of all log-transformed condition-pairs between Joystick/Response pad and congruent/incongruent.

All post-preprocessing data is depicted as cumulative distribution functions for all conditions in Fig 3. The blue and the orange (the lines that start on the very left) lines correspond to the button press conditions, the purple and the yellow (the lines that start more to the right) lines correspond to the joystick condition. The figure indicates that button press trials are generally faster than joystick trials, as both button press conditions begin to grow significantly earlier and reach their inflection point sooner than the joystick conditions. Additionally, the button press conditions look very similar in their course. However, the joystick conditions do differ in slope. Here, the yellow (the brighter) line that corresponds to the congruent joystick condition shows a steeper slope than the incongruent joystick condition. A steeper slope of a cumulative distribution function indicates that the variance of reaction times is smaller in the respective condition. Those observations allow for a first pre-analysis interpretation of the data, which suggests that participants were less hesitant in reacting to congruent conditions when using a 


\section{EMBODIEMENT OF THE AUTOMATIC APPROACH BIAS}

joystick, yielding a smaller variance in contrast to the incongruent joystick condition, while there seems to be no such difference for button presses.

Main effect: Device

0
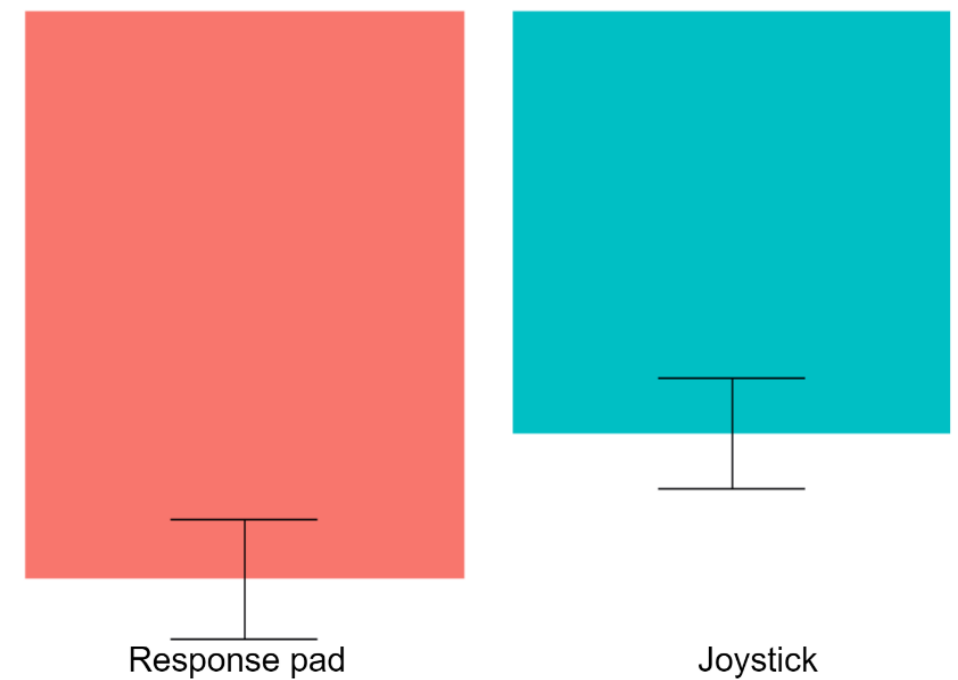

$-0.3$

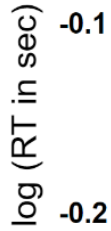

0

Main effect: Condition

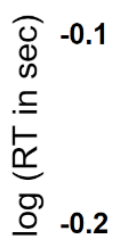

$-0.3$
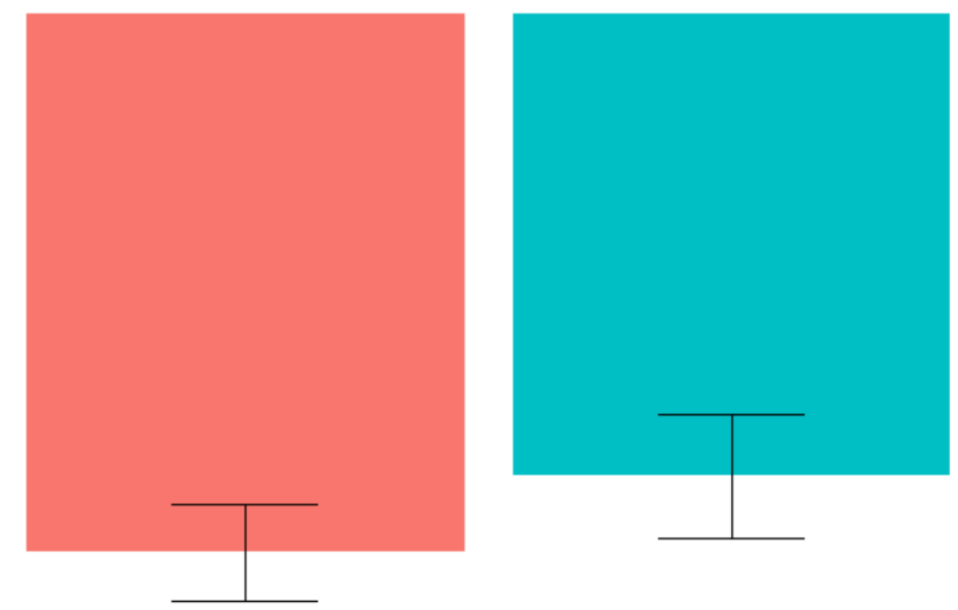

Congruent

Incongruent

Main effect: Valence

0

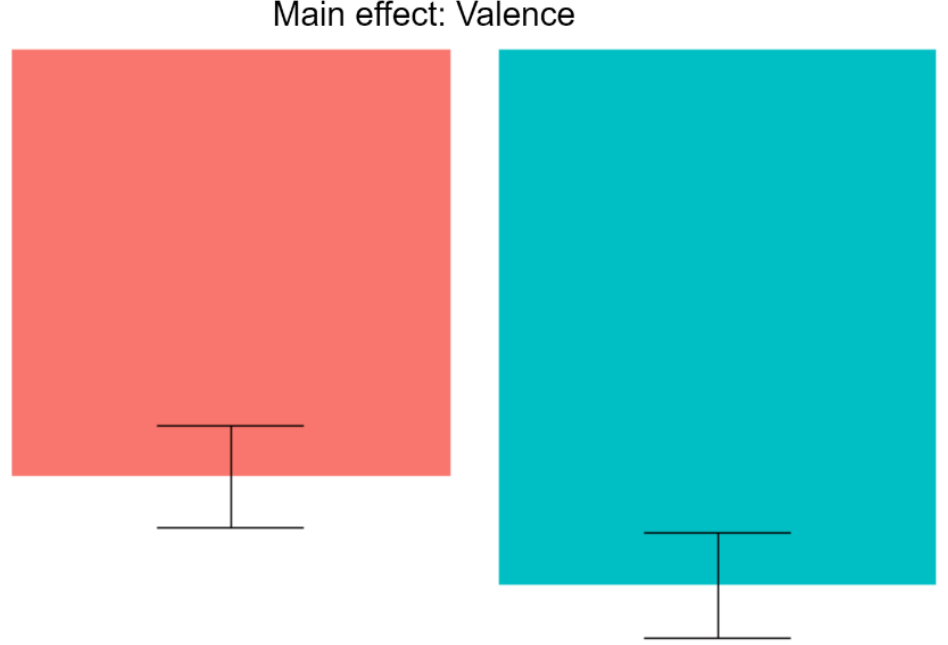

$-0.3$

Negative

Positive

Fig. 4 Main effects for device (joystick vs response pad), condition (congruent vs. incongruent) and valence (positive vs. negative) 


\section{EMBODIEMENT OF THE AUTOMATIC APPROACH BIAS}

\section{Main effects and interaction}

Since our main hypothesis concerned the embodiedness of the automatic approach bias, we focused our analysis on the comparison of the reaction time differences between conditions dependent on the devices used. For all purposes we used linear mixed models (LMM) to analyse reaction times. The LMMs were calculated with the fitlme function of matlab fit by restricted maximum likelihood estimation (REML). Degrees of freedom were assumed to be constant and equal to $n-p$, where $n$ is the number of observations and $p$ is the number of fixed effects (residual method). For the calculation of effect sizes, we used Cohens d. For that we calculated the standard-deviation from the standard-error of the model and we used the specific betas of the model as difference between means. The calculation procedure was the same for all effects. In our LMM we modelled reaction times by condition, device and valence as fixed effects and interactions between them. As random effect, we used random intercepts for the grouping variable subject. For all predictors, we used an effect coding scheme with binary factors coded as -0.5 and 0.5 . The advantage of this coding scheme is that the fixed effect intercept is estimated as the grand average across all conditions and not as a baseline condition average. Thus, the resulting estimates can be directly interpreted as the main effects. Beside the main effects for "device", "condition" and "valence", for our main hypothesis the interaction between the fixed effects "device" and "condition" was of special interest.

First, we found a significant main effect for the fixed effect 'condition' (congruent vs. incongruent). The effect was significant with $\left(\mathrm{t}(7225)=-6.5301, \mathrm{p}<7 * 10^{-11}\right)$ (Figure 4a). The logtransformed reaction time for the incongruent condition was about 0.038 times higher when compared to the congruent condition ( $\beta=-0.0375)$. This corresponds to a percentage-increase in reaction times of $3.9 \%$, meaning participants reacted faster in the congruent condition compared to the incongruent condition by a factor of $3.9 \%$. We calculated an effect size of $d=0.953$ for this effect ( $\mathrm{SE}=0.0055)$, which is a large effect-size according to (Cohen 1988). Averaged over both devices' participants were $41.8 \mathrm{~ms}$ faster when responding to congruent conditions in comparison to incongruent conditions. Thus, 


\section{EMBODIEMENT OF THE AUTOMATIC APPROACH BIAS}

it can be concluded, that participants were overall faster in responding to congruent than to incongruent trials.

Furthermore, we also found a significant main effect for the fixed effect 'device' (joystick vs. button press). Here, the effect size was significant with $\left(\mathrm{t}(7225)=14.714, \mathrm{p}<2 * 10^{-48}\right)$ (Figure $\left.4 \mathrm{~b}\right)$. The log-transformed reaction time for button presses on the response pad was about 0.085 times smaller than for the joystick condition $(\beta=0.0845)$. This corresponds to an increase of $8.1 \%$ between devices, meaning participants were $8.1 \%$ faster for button press trials compared to joystick trials. We calculated Cohens $d$ for the main effect 'device' with $d=2.1462(\mathrm{SE}=0.0057)$, which corresponds to a huge effect size (Cohen 1988). Here, averaged over both conditions' participants were $58.3 \mathrm{~ms}$ slower when responding with a joystick in comparison to responding with the response pad. It can be concluded that averaged over both conditions, participants had slower reaction times for the device 'joystick' in comparison to the device 'response pad'. 


\section{EMBODIEMENT OF THE AUTOMATIC APPROACH BIAS}

Lastly, we found a significant main effect for the fixed effect 'valence' (positive vs. negative). The effect was significant with $\left(\mathrm{t}(7225)=-9.787, \mathrm{p}<10^{-22}\right)($ Figure $4 \mathrm{c})$. The log-transformed response time for negative pictures was about 0.056 times smaller, than for positive pictures $(\beta=-0.0562)$. This corresponds to a reaction time increase of $5.47 \%$ between positive and negative pictures. Thus, participants were $5.47 \%$ faster in responding to negative pictures compared to responding to positive pictures. Cohens $d$ for the main effect 'valence' was calculated with $d=1.4275)(\mathrm{SE}=0.0057)$, which is a huge effect size (Cohen 1988). It can be concluded that participants were significantly faster in reacting to negative stimuli than in reacting to positive stimuli.

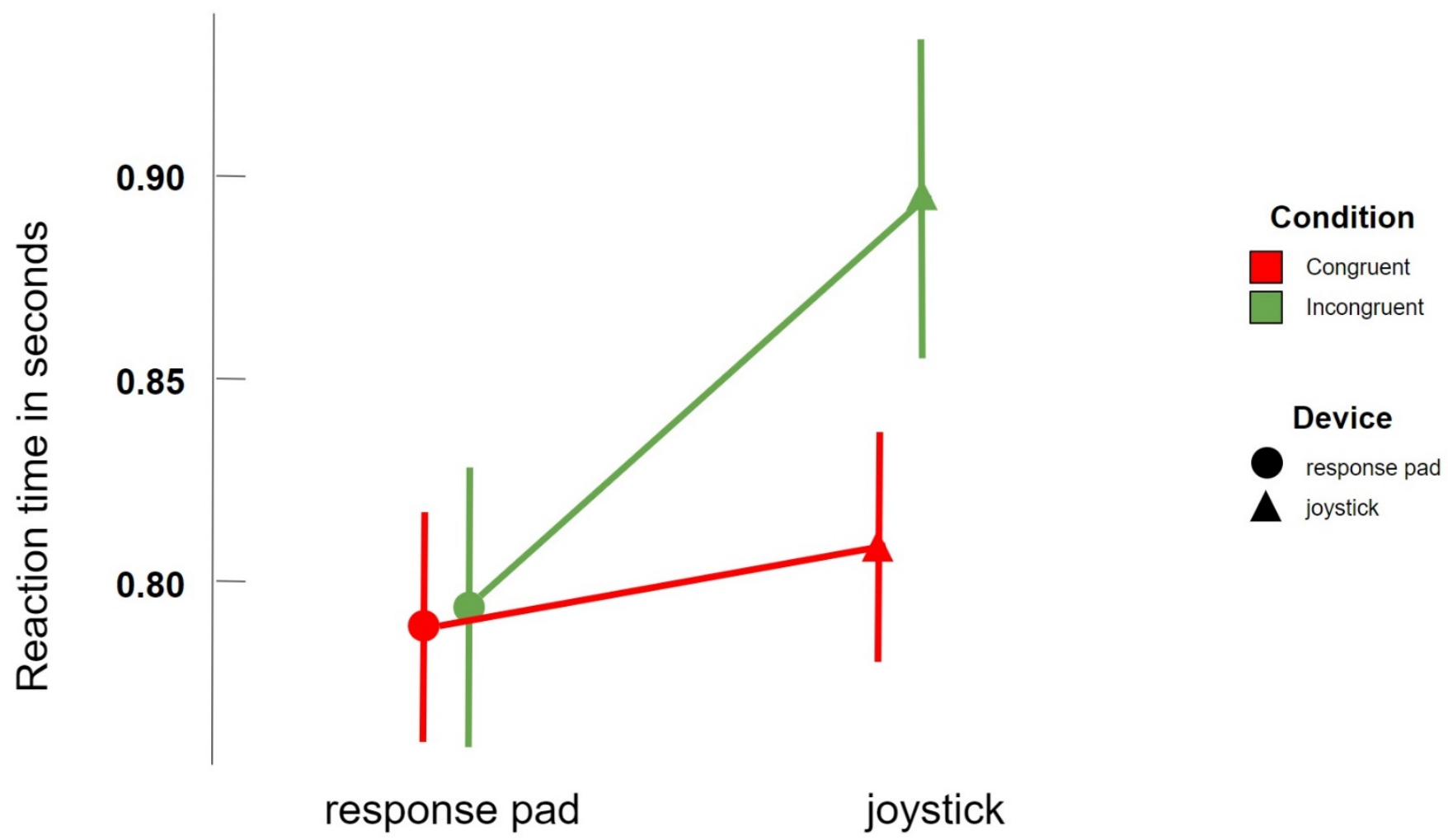

Fig. 5 Interaction between condition and device. Participants were significantly slower for the incongruent condition when using the joystick but not when using the response pad.

The only significant interaction we found, was the interaction between the fixed effects 'device' and 'condition' (Fig 5). This interaction was significant with $\left(\mathrm{t}(7225)=-7.1619, \mathrm{p}<8^{*} 10^{-13}\right)$. All other interactions were tested, but no other two or three-way interaction was significant (all $p>0.28$ ). The difference in reaction time between congruent and incongruent condition was larger for the joystick 


\section{EMBODIEMENT OF THE AUTOMATIC APPROACH BIAS}

than for the button press by a factor of 19.4. The difference in reaction time between joystick and button press was larger for incongruent conditions than for congruent conditions by a factor of 4.7 . Consequently, the longest average reaction time was observed for the incongruent joystick condition. Cohens $\mathrm{d}$ for the interaction 'device : condition' was calculated with $\mathrm{d}=1.0447(\mathrm{SE}=0.011)$, which still is a huge effect size (Cohen 1988). As Fig. 5 displays, the Interaction between the parameters device and condition explains a lot of the main effects. While button presses are generally faster than joystick trials and congruent trials are generally faster than incongruent trials, it really is the incongruent joystick condition that makes the difference. The interaction shows that incongruent trials are significantly slower than congruent trials when using a joystick, but there is merely a difference between them when using a response pad.

\section{Discussion}

In the present study we tried to resolve the problem that despite its theoretical and clinical potential, an embodied take on the automatic approach bias seems hard to sustain if such a bias is present in a setup that requires no significant bodily behavior to begin with (Fiedland and Wiers 2018). Therefore, we conducted a systematic comparison between two different approach-avoidance tasks, one using button presses and one using a joystick. By this we aimed to investigate whether the gesture in the sense of an actual bodily movement influences - or might even be necessary for the effect. In line with previous research we expected to find a main effect for the joystick (Phaf et al. 2014) and for the button press condition (Peeters et al. 2012). Due to recent considerations of the embodied paradigm regarding approach and avoidance gestures (Fridland and Wiers 2018) we also hypothesized that the effect would be more pronounced for the joystick than for the button press, and that this difference could be explained by the difference in device. We found an automatic approach bias for the joystick, which was to be expected, since it was shown many times that this 'most classic' version of an approachavoidance task yields an automatic approach bias. We could however not reproduce the same effect for a button press. The fact that a button press approach-avoidance task did not yield the same effect can be considered evidence that an automatic approach bias is in fact not present - or at least not that strong 


\section{EMBODIEMENT OF THE AUTOMATIC APPROACH BIAS}

- if the corresponding gesture is missing. This suggests that the difference in device is crucial for an automatic approach bias to be present and since the main difference between devices is supposed to be the presence of a (significant) bodily gesture, the automatic approach bias might therefore in fact be grounded in the bodily movement of approach and avoidance.

The following discussion concerns the interpretation of the different main effects we found (valence, device, condition) and of the significant interaction between device and condition. We found strong main effects for all fixed variables (valence, condition, device). With those findings we reproduced important known effects. First, we reproduced the finding that negative stimuli are generally faster responded to than positive stimuli. This can be explained by the evolutionary necessity to act faster when confronted with something negative (i.e. dangerous) and confirms theories about different pathways of processing (e.g. Lang et al., 1990). Second, we reproduced the general effect of an approach avoidance bias, meaning subjects being faster in approaching positive stimuli and avoiding negative stimuli than vice versa (e.g. Czeszumski 2020). This reproduces the main findings of the field and confirms that humans seem in fact faster in approaching positive stimuli (i.e. things they want or like) and avoiding negative stimuli (i.e. stimuli that disgust them, seem dangerous or are dislikeable) than vice versa. Third, we found a main effect for device, that suggests that button presses are generally faster than joystick trials. And although the action as such might just be quicker, those results have to be interpreted in the context of the interaction between device and condition. As Fig. 5 shows, both main effects (device and condition) are mostly explained by the strong divergence between congruent and incongruent trials for the joystick task. This shows that participants were slower for incongruent trials only if they used a joystick, but not when they used the response pad. Since the major difference between those devices is supposed to be the involved gesture, it can be concluded that the gesture does indeed play a crucial role in how the bias originates. That the automatic approach bias is more complex than originally thought, was already pointed out by Phaf (2014), who emphasized the role of different types of instruction and context. With the described findings and the resulting importance of the involved gesture, this study adds another layer to the complexity of automatic tendencies of approach and avoidance. 


\section{EMBODIEMENT OF THE AUTOMATIC APPROACH BIAS}

Although Peeters et al. (2012) did find an automatic approach bias only using button presses, we could not reproduce those findings. This could have several reasons. First, it could be the case that an automatic approach bias for button presses is just weaker than for joystick tasks. In that case, a too small sample size could have hindered us in finding the effect. If this was the case, our results would still show that a supportive gesture potentiates the effect. Second, it is conceivable that there simply is no effect for button press approach-avoidance tasks and that the former findings could not be reproduced due to the nonexistence of the effect. In this scenario, the gesture would not only potentiate an existent effect, but would be necessary for its existence. Third, we might have been unable to reproduce the effect due to other differences in the setup. For example, as Phaf (2014) already suggested, the role of affect in this context is yet unclear. Peeters et al. (2012) were working with alcohol-dependent patients. Thus, it might be the case that the general effect is stronger in psychopathological contexts in contrast to working with healthy subjects. If and how exactly such an effect modifies the automatic approach bias has yet to be shown in further research. It can be concluded that while it seems undeniable that the gesture plays a role for an automatic approach bias, it is not clear yet how it exactly contributes to the bias, i.e., whether it only potentiates an existent effect or is necessary for the effect's existence.

We demonstrated that the gesture - the movement of the arm away or towards our body - in a situation of approach and avoidance matters. The following section will focus on what this means for theories of embodiment. If we push something away, we do not want it to be close to us, we avoid it; if we move something towards our body, be it something to eat or a person that we want to hug, this is a movement of approach. The movement of our arm - the gesture that we use to approach or to avoid certain things in our environment - supports our action. The observation that certain compatibility effects - like the automatic approach/avoidance bias - are only present if combined with a corresponding gesture suggests that there is more to the gesture than it just being a part of the process of approach and avoidance. It suggests that the gesture is part of what it means to approach or avoid something as Gallagher (2005) and many other authors have claimed. In this sense the automatic approach bias is embodied, possibly in one of the ways that Fridland and Wiers (2018) suggested. 


\section{EMBODIEMENT OF THE AUTOMATIC APPROACH BIAS}

Yet, there is more to gestures. While the idea that pushing something away from us means avoidance and pulling something towards us means approach, this cannot be the full story. If one gets scarred and withdraws his/her hand from a spider this is a movement towards the body while at the same time being an avoidance movement. Another factor to consider is the reference frame. A joystick movement does not necessarily correspond to a natural movement. If one pushes the joystick away, that might as well be interpreted as a movement of their own body into the same direction (as for example in videogames, when controlling an avatar). For, example, in manikin-tasks or modified approach avoidance tasks, corresponding biases have been found, if the context was different for the participants. Thus, it seems that tendencies of approach and avoidance and their corresponding gestures are contextsensitive (e.g. Zhang et al. 2012; Markman and Brendl 2005). Further research might show how context sensitivity influences the embodiment of the automatic approach bias.

As our data suggests, the automatic approach bias is - most likely - embodied. Besides theoretical insight into the mechanisms of human approach/avoidance behavior, this might also have severe clinical implications. The automatic approach bias can be modified by means of implicit approach avoidance tasks over a longer period of time, so-called 'cognitive bias modifications'. This is especially important for individuals suffering from substance use disorders. For those, but also for other psychological disorders such as depression, it has been shown that a modification of people's automatic approach bias f.e. towards cues that show the desired substance has a profound impact on their success of treatment, craving rates and subconscious action tendencies (Lindenmeyer 2019; MacLeod and Mathews 2012; Mehl et al. 2019; Mühlig et al. 2016; Rabinovitz and Nagar 2015; C. E. Wiers et al. 2015). And while those outcomes already seem impressive, they get a new dimension when thinking about the fact that the corresponding cognitive bias modifications have all been performed with a joystick only. Taking into account that it is very likely that the gesture itself has an influence on the strength of the effect, this leads to the following conclusion: If even a relatively 'weak' gesture like a joystick movement - that indeed is associated with approach and avoidance but far from really incarnating the concepts - can have such an enormous effect, how potent must the possibilities for treatment be if the procedure is improved by enlarging the immersion or choosing stronger gestures to 
EMBODIEMENT OF THE AUTOMATIC APPROACH BIAS

perform the training with? It might be possible to potentiate the success of such treatments by choosing gestures that really are incarnating the concepts. That might for instance be to smash the cue with a bat for avoidance. The corresponding cognitive bias modifications could be implemented into VR to additionally increase the immersion. Approaches to embodied addiction such as Miller (2020) might complement and complete the picture and give important evidence for further directions of research. Of course, a lot of questions have to be answered before such an embodied approach to clinical applications can actually be conducted, but promising trials like Eiler et al. (2019), who could demonstrate a first success of the concept for smokers, ensure the potential and the worth of investigating into this direction.

\section{Conflict of Interest Statement}

The authors declare that the research was conducted in the absence of any commercial or financial relationships that could be construed as a potential conflict of interest.

\section{Acknowledgments}

This project was funded by the Deutsche Forschungsgemeinschaft (DFG, German Research Foundation) - project number GRK-2185/1 (DFG Research Training Group Situated Cognition). We also gratefully acknowledge the support of the Research Training Group Situated Cognition (RTG SC at https://situated-cognition.com/). 


\section{EMBODIEMENT OF THE AUTOMATIC APPROACH BIAS}

\section{References}

Booth, C., Spronk, D., Grol, M., \& Fox, E. (2018). Uncontrolled eating in adolescents: The role of impulsivity and automatic approach bias for food. Appetite, 120, 636-643.

Bradley, B. P., Field, M., Healy, H., \& Mogg, K. (2008). Do the affective properties of smokingrelated cues influence attentional and approach biases in cigarette smokers? Journal of Psychopharmacology , 22(7), 737-745.

Chen, M., \& Bargh, J. A. (1999). Consequences of Automatic Evaluation: Immediate Behavioral Predispositions to Approach or Avoid the Stimulus. Personality \& Social Psychology Bulletin, 25(2), 215-224.

Clark, A. (1998). Being There: Putting Brain, Body, and World Together Again. MIT Press.

Cohen, J. (1988). Statistical power analysis for the behavioral sciences (Revised ed.). Hillsdale, NJ: Lawrence Earlbaum Associates. Inc.

Cousijn, J., Goudriaan, A. E., \& Wiers, R. W. (2011). Reaching out towards cannabis: approachbias in heavy cannabis users predicts changes in cannabis use: Approach-bias and cannabis use. Addiction , 106(9), 1667-1674.

Czeszumski, A., Albers, F., Walter, S., \& König, P. (2020). Let Me Make You Happy, And I' 11 Tell You How you look around: Using an Approach-Avoidance Task as an Embodied Emotion Prime in a Free-Viewing Task. bioRxiv. https://www.biorxiv.org/content/10.1101/2020.09.09.289249v2.abstract

Dickson, H., Kavanagh, D. J., \& MacLeod, C. (2016). The pulling power of chocolate: Effects of approach-avoidance training on approach bias and consumption. Appetite, 99, 46-51. 
EMBODIEMENT OF THE AUTOMATIC APPROACH BIAS

Eiler, T. J., Grünewald, A., \& Brück, R. (2019). Fighting Substance Dependency Combining AAT Therapy and Virtual Reality with Game Design Elements. VISIGRAPP (2: HUCAPP), 2837.

Engel, A. K., Maye, A., Kurthen, M., \& König, P. (2013). Where's the action? The pragmatic turn in cognitive science. Trends in Cognitive Sciences, 17(5), 202-209.

Ernst, L. H., Plichta, M. M., Dresler, T., Zesewitz, A. K., Tupak, S. V., Haeussinger, F. B., Fischer, M., Polak, T., Fallgatter, A. J., \& Ehlis, A.-C. (2014). Prefrontal correlates of approach preferences for alcohol stimuli in alcohol dependence. Addiction Biology, 19(3), 497-508.

Fodor, J. A. (1975). The Language of Thought. Harvard University Press.

Fodor, J. A. (1981). Representations: Philosophical essays on the foundations of cognitive science. Mit Press Cambridge, MA.

Fox, E., Zougkou, K., Ashwin, C., \& Cahill, S. (2015). Investigating the efficacy of attention bias modification in reducing high spider fear: The role of individual differences in initial bias. Journal of Behavior Therapy and Experimental Psychiatry, 49(Pt A), 84-93.

Fridland, E., \& Wiers, C. E. (2018). Addiction and embodiment. Phenomenology and the Cognitive Sciences, 17(1), 15-42.

Gallagher, S. (2006). How the Body Shapes the Mind. Clarendon Press.

Goldman, A. I. (2013). A Moderate Approach to Embodied Cognitive Science. In Joint Ventures (pp. 233-256). https://doi.org/10.1093/acprof:osobl/9780199874187.003.0011 
EMBODIEMENT OF THE AUTOMATIC APPROACH BIAS

Hertel, P. T., \& Mathews, A. (2011). Cognitive Bias Modification: Past Perspectives, Current Findings, and Future Applications. Perspectives on Psychological Science: A Journal of the Association for Psychological Science, 6(6), 521-536.

Heuer, K., Rinck, M., \& Becker, E. S. (2007). Avoidance of emotional facial expressions in social anxiety: The Approach-Avoidance Task. In Behaviour Research and Therapy (Vol. 45, Issue 12, pp. 2990-3001). https://doi.org/10.1016/j.brat.2007.08.010

Jeromin, F., Nyenhuis, N., \& Barke, A. (2016). Attentional bias in excessive Internet gamers: Experimental investigations using an addiction Stroop and a visual probe. Journal of Behavioral Addictions, 5(1), 32-40.

Kakoschke, N., Hawker, C., Castine, B., de Courten, B., \& Verdejo-Garcia, A. (2018). Smartphone-based cognitive bias modification training improves healthy food choice in obesity: A pilot study. European Eating Disorders Review: The Journal of the Eating Disorders Association, 26(5), 526-532.

Kaspar, K., Gameiro, R. R., \& König, P. (2015). Feeling good, searching the bad: Positive priming increases attention and memory for negative stimuli on webpages. Computers in Human Behavior, 53, 332-343.

Kleiner, M., Brainard, D., \& Pelli, D. (2007). What's new in Psychtoolbox-3? https://pure.mpg.de/rest/items/item_1790332/component/file_3136265/content

Koster, E. H. W., \& Hoorelbeke, K. (2015). Cognitive bias modification for depression. In Current Opinion in Psychology (Vol. 4, pp. 119-123). https://doi.org/10.1016/j.copsyc.2014.11.012

Lang, P. J., Bradley, M. M., \& Cuthbert, B. N. (1990). Emotion, attention, and the startle reflex. Psychological Review, 97(3), 377-395. 
EMBODIEMENT OF THE AUTOMATIC APPROACH BIAS

Lang, P. J., Bradley, M. M., \& Cuthbert, B. N. (1997). International affective picture system (IAPS): Technical manual and affective ratings. NIMH Center for the Study of. https://www2.unifesp.br/dpsicobio/adap/instructions.pdf

Lindenmeyer, J. (2019). Cognitive Bias Modification zur Rückfallprävention bei Alkoholabhängigkeit: In Deutscher Suchtkongress 2019. https://doi.org/10.1055/s-00391696229

MacLeod, C., \& Mathews, A. (2012). Cognitive bias modification approaches to anxiety. Annual Review of Clinical Psychology, 8, 189-217.

Markman, A. B., \& Miguel Brendl, C. (2005). Constraining Theories of Embodied Cognition. In Psychological Science (Vol. 16, Issue 1, pp. 6-10). https://doi.org/10.1111/j.09567976.2005.00772.x

Marr, D. (1982). Vision: A Computational Investigation into the Human Representation and Processing of Visual Information. http://papers.cumincad.org/cgi-bin/works/Show?fafa

Mehl, N., Morys, F., Villringer, A., \& Horstmann, A. (2019). Unhealthy yet Avoidable- - How Cognitive Bias Modification Alters Behavioral and Brain Responses to Food Cues in Individuals with Obesity. In Nutrients (Vol. 11, Issue 4, p. 874). https://doi.org/10.3390/nu11040874

Miller, M., Kiverstein, J., \& Rietveld, E. (2020). Embodying addiction: A predictive processing account. Brain and Cognition, 138, 105495.

Mühlig, S., Paulick, J., Lindenmeyer, J., Rinck, M., Cina, R., \& Wiers, R. W. (2016). Applying the "Cognitive Bias Modification" concept to smoking cessation - A Systematic Review. In SUCHT (Vol. 62, Issue 6, pp. 333-354). https://doi.org/10.1024/0939-5911/a000454 
EMBODIEMENT OF THE AUTOMATIC APPROACH BIAS

Peeters, M., Wiers, R. W., Monshouwer, K., van de Schoot, R., Janssen, T., \& Vollebergh, W. A. M. (2012). Automatic processes in at-risk adolescents: the role of alcohol-approach tendencies and response inhibition in drinking behavior: Alcohol use in at-risk adolescents. Addiction , 107(11), 1939-1946.

Phaf, R. H., Mohr, S. E., Rotteveel, M., \& Wicherts, J. M. (2014). Approach, avoidance, and affect: a meta-analysis of approach-avoidance tendencies in manual reaction time tasks. Frontiers in Psychology, 5, 378.

Rabinovitz, S., \& Nagar, M. (2015). Possible end to an endless quest? Cognitive bias modification for excessive multiplayer online gamers. Cyberpsychology, Behavior and Social Networking. https://www.liebertpub.com/doi/abs/10.1089/cyber.2015.0173

Rinck, M., \& Becker, E. S. (2007). Approach and avoidance in fear of spiders. Journal of Behavior Therapy and Experimental Psychiatry, 38(2), 105-120.

Schroeder, P. A., Lohmann, J., Butz, M. V., \& Plewnia, C. (2016). Behavioral Bias for Food Reflected in Hand Movements: A Preliminary Study with Healthy Subjects. Cyberpsychology, Behavior and Social Networking, 19(2), 120-126.

Stevens, E. S., Behar, E., \& Jendrusina, A. A. (2018). Enhancing the Efficacy of Cognitive Bias Modification for Social Anxiety. Behavior Therapy, 49(6), 995-1007.

Strack, F., Martin, L. L., \& Stepper, S. (1988). Inhibiting and facilitating conditions of the human smile: a nonobtrusive test of the facial feedback hypothesis. Journal of Personality and Social Psychology, 54(5), 768-777.

Tendolkar, I., Vrijsen, J. N., \& Becker, E. S. (2019). „Cognitive bias modification“ als mögliche Add-on-Therapie bei Depression: Stand der Forschung. Psychotherapeut, 64(3), 180-185. 
EMBODIEMENT OF THE AUTOMATIC APPROACH BIAS

Wiers, C. E., Kühn, S., Javadi, A. H., Korucuoglu, O., Wiers, R. W., Walter, H., Gallinat, J., \& Bermpohl, F. (2013). Automatic approach bias towards smoking cues is present in smokers but not in ex-smokers. Psychopharmacology, 229(1), 187-197.

Wiers, Corinde E., Christine Stelzel, Thomas E. Gladwin, Soyoung Q. Park, Steffen Pawelczack, Christiane K. Gawron, Heiner Stuke, et al. 2015. "Effects of Cognitive Bias Modification Training on Neural Alcohol Cue Reactivity in Alcohol Dependence.” The American Journal of Psychiatry 172 (4): 335-43.

Wiers, C. E., Stelzel, C., Park, S. Q., Gawron, C. K., Ludwig, V. U., Gutwinski, S., Heinz, A., Lindenmeyer, J., Wiers, R. W., Walter, H., \& Bermpohl, F. (2014). Neural correlates of alcohol-approach bias in alcohol addiction: the spirit is willing but the flesh is weak for spirits. Neuropsychopharmacology: Official Publication of the American College of Neuropsychopharmacology, 39(3), 688-697.

Wiers, R. W., Eberl, C., Rinck, M., Becker, E. S., \& Lindenmeyer, J. (2011). Retraining Automatic Action Tendencies Changes Alcoholic Patients’ Approach Bias for Alcohol and Improves Treatment Outcome. In Psychological Science (Vol. 22, Issue 4, pp. 490-497). https://doi.org/10.1177/0956797611400615

Wiers, R. W., Rinck, M., Dictus, M., \& van den Wildenberg, E. (2009). Relatively strong automatic appetitive action-tendencies in male carriers of the OPRM1 G-allele. Genes, Brain, and Behavior, 8(1), 101-106.

Zhang, Y., Proctor, R. W., \& Wegener, D. T. (2012). Approach-avoidance actions or categorization? A matching account of reference valence effects in affective S-R compatibility. Journal of Experimental Social Psychology, 48(3), 609-616. 


\section{EMBODIEMENT OF THE AUTOMATIC APPROACH BIAS}

Zhou, Y., Li, X., Zhang, M., Zhang, F., Zhu, C., \& Shen, M. (2012). Behavioural approach tendencies to heroin-related stimuli in abstinent heroin abusers. Psychopharmacology, 221(1), $171-176$.

Zhou, Z., Yuan, G., \& Yao, J. (2012). Cognitive biases toward Internet game-related pictures and executive deficits in individuals with an Internet game addiction. PloS One, 7(11), e48961. 